\title{
The Delicate Dance of Teaching Rounds
}

\section{Sherine Salib ${ }^{1}$}

Published online: 16 May 2019

(C) International Association of Medical Science Educators 2019

Most will agree. This is certainly a topic that is near and dear to hospital attendings' hearts - teaching rounds and the everlasting battle to achieve everything in an ever-shortening amount of time.

I envision teaching rounds as a finely choreographed dance - everything must work together seamlessly for an effective and well-balanced end product.

How is it humanly possible to see all the patients on the infamous list, embed teaching, give feedback, engage all the various multi-levels of learners, deliver top-notch care, participate in inter-professional discussions, and concurrently maintain a high level of enthusiasm-all within a 2-hour timeframe? It is no wonder that there is a disconnect $[1,2]$ between what we all know to be the purpose of teaching rounds, and what actually happens during rounds.

There has been much discussion about various strategies, as well as debates, often accompanied by strong feelings about right and wrong ways to do things. Exploration of learner perceptions [3] of teaching rounds exposes dissatisfaction in achieving goals. Bedside rounds, for example, are gaining more traction and many are working on strategies to overcome barriers [4]..

Much of the teaching activities, however, do not occur at the bedside.

There is no consensus on how best to conduct teaching rounds, but there is more agreement on what all teaching rounds should include, while being mindful not to overly extend rounding time. We have learned some things by exploring, for instance, what "all-star" attendings do [5].

I have taken the approach of being transparent with my team. There is no absolute right or wrong way to do teaching rounds, and therefore my various teams and I have

Sherine Salib

sherine.salib@austin.utexas.edu

1 The Department of Internal Medicine, Dell Medical School at the University of Texas at Austin, Austin, TX, USA experimented with different approaches, giving and receiving feedback on what works and what does not, while trying to be nimble - and humble - enough to change, tweak, and adjust rounds based on the teams' feedback.

In this brief article, I share some little but impactful processes that we adopted, and many of which readers may want to explore incorporating, regardless of the precise structure of their teaching rounds. While not novel in themselves, together they help achieve the above-mentioned goals.

Rounding Pearls

- "Show me the way"

Set expectations $[6,7] —$ mini, daily expectations, and whole block expectations

Learners thrive when given clear expectations. So, we set two types of expectations - daily mini-expectations, and more medium-term expectations for the duration of the whole rotation.

The mini daily expectations include things such as, "team, today we will plan to do table rounds to discuss the more complex patients for 45 minutes, round to see the patients with bedside discussions for one-and-a-half hours, then sit down again to review the clinical questions that various team members were assigned and researched for us for a total of 20 minutes." The mini daily expectations may vary from day-to-day, depending on many factors. The more medium-term expectations are set for the whole time that I am with the team, typically 2 weeks at a time. These include expectations about documentation, post-discharge test follow-up, sticking together during rounds, frequent in-real-time two-way-feedback, etc.

Once expectations are set, they work both ways: I keep the team accountable, and the team keeps me accountable. I make this clear, and revisit these bi-directional expectations frequently.

- "Keep me interested"

Change things up intermittently

I have experimented with different rounding styles and formats over the years, taking into consideration my 
learners' feedback and peer observation. I believe I have found a system that works well most of the time, but also try to be flexible and open to trying new formats. Even with a system that works, the team and I change things up intermittently during rounds. In addition to taking into account the particulars of each day (e.g., patient census, new sick patients), team dynamics, as well as team strengths and weaknesses, changing the routine intermittently keeps the team on their toes, and constantly engaged, but most importantly, it allows the rounding experience to be tailored to the individual team. Consistency is good, but shaking up the routine every now and then is important.

- “Keep us all engaged, not just the presenter" [8]

Real-time research using team members who are not presenting

We often start rounds with approximately $45 \mathrm{mi}$ nutes of "table rounds" which allows us to discuss some of the more complex patients sitting around a table, computers open, everyone present, everyone engaged, and where we can all hear each other clearly. Inevitably, questions come up that we do not know the answers to. Our "real-time researchers", often-but not always - the student on the team who is not presenting at the time do spot-research if the question lends itself to a brief review, which many miniclinical questions do. This beats accumulating many clinical questions that the team never has the time to research in detail at a later point (although there is a time and place for those.)

- "Encourage our curiosity, but do not overload us" [9]

Patient-specific mini-clinical questions

Part of the expectation setting is discussing how we will handle clinical questions as they come up in patient care. I ask the team members to each identify a clinical question regarding their patients' care. Once identified, this team member does a 15-minute literature review around that question later in the day, and subsequently takes 3-5 minutes to share this with the team the next day. Typically, students are assigned a daily mini-clinical question, interns a couple of questions each week, and upper-level residents a single more involved clinical question per week.

By setting clear expectations on what is expected from the mini-clinical question review, learners do not find the process overwhelming; the team learns and patient care is improved. The mini-clinical question needs to be patient-specific and preferably on a learner-identified topic. Learner-identified questions, as opposed to educator-identified questions, serve better in kindling a spirit of inquiry, and selfdirected learning.
- "Please be efficient. You know how much we have to do!" [10]

Embed required activities into the flow of rounds, e.g. mini-CEX [11]

There are many required activities that help the educator gauge the clinical performance of the learner. Most are naturally embedded in teaching rounds, such as verbal presentations and clinical reasoning. However, other assessments, such as the "mini-CEX" or "mini-clinical examination", (a formative assessment tool of core clinical skills), are frequently conducted at another time outside teaching rounds. I would suggest that teaching rounds, in fact, lend themselves nicely to incorporating the mini-CEX intermittently. For instance, you may ask an intern or resident to counsel one of their patients regarding the updated plan of care, or demonstrate part of a physical exam to the rest of the team. Individualized feedback should be shared with the learner that same day.

- "Help me understand the method behind the madness"

RIME [12] — use it and help them understand whytailor its use to each learner

When a new team and I start to work together, we briefly go through the RIME methodology of teaching (reporter, interpreter, manager, educator) which was developed by Dr. Louis Pangaro. I tell that them that, depending on the time of the year, and the level of each learner, we will typically be concentrating on a couple of aspects of RIME. For instance, for the 2nd-year resident, we are usually working on interpretation and management, but also intermittently checking that they have their " $R$ " right. We discuss that reporting is not as easy as it sounds, since each hospitalized patient typically has, at a guess, more than 500 data points at any given time, let alone an extensive history.

- "Be my safety net"

"Trust but verify"

I share with my team that I review the patients' data ahead of rounds, but that they are, nonetheless, fully responsible for knowing their patients. They understand that these are their patients and I am just there for support. I do this because I am their "safety net". I make sure they understand the reasoning, and I find that they appreciate this. We discuss James Reason's "Swiss cheese" [13] model of patient safety, and the multiple layers necessary to plug the holes, to go from Swiss cheese to havarati [14]! However, we agree at the beginning of the rotation that, if at any point, I know more about their patients than they do, then they do not know their patients well enough. We also discuss that by us all agreeing to review the data thoroughly prior to rounds, the team can concentrate less on the "R", and more on the "I" and "M" of RIME. 
- "Would you do a little leg-work on your own please?"

Residents frequently have concerns about lack of teaching due to the clinical volume [15]. This is a real concern, agreed upon by both learners and educators. The highly debated concept of "service over education" as perceived by residents entails not only indirect patient care, but some direct patient care activities as well. As alluded to above, it is sometimes physically impossible to accomplish all the agreed-upon goals of rounds without prolonging rounds and eating into the protected time for conferences, and afternoon admissions and discharges. Despite many creative attempts to reconfigure rounds, at times, the only possible way to accomplish everything is for the attending physician to put in some "leg work" on their own time. Personally, I have found it most effective to see some of the more chronic patients on my own prior to teaching rounds. The team and I still discuss those patients' care together, and I always offer to go back and see those patients with the team should there be any concerns. Unanimously, the residents have been grateful for this gift of time, and for the opportunity not to rush through rounds just to see everyone together every single day. I do make a point, though, of ensuring that we visit each patient on the list together as team at least every other day.

- "How am I doing?"

Constant feedback

There is no doubt that feedback is pivotal to the continuing growth of learners. The literature shows that constant, real-time feedback is one of the top determinants in who is considered an "all-star" clinical teacher [5].

But how is feedback incorporated into rounds? Like micro-teaching, micro-feedback is highly effective and happens more often than learners - and even teachersrealize. Going back to expectation setting, we briefly discuss the approach to feedback when the team meets on day 1. All team members have one-on-one, sit-down feedback with me as the attending prior to leaving the rotation, both at the mid-point and the end of the rotation. I also let the team know to expect a lot of "micro-feedback" during our work together - as we step out of a patient's room, take the stairs, or walk from room to room-frequent and diverse micro-feedback, perhaps on a presentation they just gave or their bedside manner. I label feedback as such, and set specific expectations on how to continue or change the discussed the discussed behavior.

- "Focus our learning \& help us remember"

The end-of-rounds "brain squeeze": "one thing I learned today"

I have found that after attending a lecture, or reading an article, asking myself the question "what is one thing I will remember from this?" really helps me concentrate and take away at least this one thing from that experience. I have adopted the same practice with my learners during attending rounds. At the end of rounds, I hand each of them a small "post-it" sized piece of paper and ask them to write "one thing I learned today". It can be a medical knowledge item, a practical matter - anything. The act of thinking through what they learned, picking one item, and then taking pen to paper to write that, consolidates the learning [16].

Sometimes, we share what we have learned, and sometimes we revisit these learning points later in the week. My learners tell me they usually remember these things long after our weeks of working together are over. I do too.

- "You are my role model!"

Keep up that enthusiasm [17]!

As simple as this sounds, this may be the most important point of all. As the attending on the team, you set the "tone". Studies have shown that a clinical teacher's enthusiasm and non-cognitive abilities are just as important as their cognitive abilities. So, what exactly should the teacher maintain enthusiasm for - patient care, teaching, medicine, the team's work? The short answer is everything.

\section{Compliance with Ethical Standards}

Conflict of Interest The author declares that she has no conflict of interest. Thank you.

\section{References}

1. Hulland O, Farnan J, Rabinowitz R, Kearns L, Long M, Monash B, et al. What's the purpose of rounds? A qualitative study examining the perceptions of faculty and students. J Hosp Med. 2017;12(11): 892-7. https://doi.org/10.12788/jhm.2835.Epub2017Sep20.PMID: 29091976.

2. Stickrath C, Noble M, Prochazka A, Anderson M, Griffiths M, Manheim J, et al. 1. Attending rounds in the current era: what is and is not happening. JAMA Intern Med. 2013;173(12):1084-9. https://doi.org/10.1001/jamainternmed.2013.6041.PMID: 23649040.

3. Rabinowitz R, Farnan J, Hulland O, Kearns L, Long M, Monash B, et al. Rounds today: a qualitative study of internal medicine and pediatrics resident perceptions. J Grad Med Educ. 2016;8(4):52331.

4. Gonzalo JD, Heist BS, Duffy BL, Dyrbye L, Fagan MJ, Ferenchick $\mathrm{G}$, et al. Identifying and overcoming the barriers to bedside rounds: a multicenter qualitative study. Acad Med. 2014;89(2):326-34. https://doi.org/10.1097/ACM.0000000000000100.

5. Merritt FW, Noble MN, Prochazka AV, Aagaard EM, Stickrath CR. Attending rounds: what do the all-star teachers do? Med Teach. 2017;39(1):100-4. https://doi.org/10.1080/0142159X.2017. 1248914.

6. Chen PH, Scanlon MH. Teaching radiology trainees from the perspective of a millennial. Acad Radiol. 2018. https://doi.org/10. 1016/j.acra.2018.02.008.

7. Gonzalo JD, Heist BS, Duffy BL, Dyrbye L, Fagan MJ, Ferenchick $\mathrm{G}$, et al. The art of bedside rounds: a multi-center qualitative study of strategies used by experienced bedside teachers. J Gen Intern Med. 2013;28(3):412-20. https://doi.org/10.1007/s11606-0122259-2. 
8. Certain LK, Guarino AJ, Greenwald JL. Effective multilevel teaching techniques on attending rounds: a pilot survey and systematic review of the literature. Med Teach. 2011;33(12):e644-50. https:// doi.org/10.3109/0142159X.2011.610844 Review.

9. Goldie J, Dowie A, Goldie A, Cotton P, Morrison J. What makes a good clinical student and teacher? An exploratory study. BMC Med Educ. 2015;15:40. https://doi.org/10.1186/s12909-015-0314-5.

10. Wang FY. Addressing resident concerns about rounding without extending rounds. J Grad Med Educ. 2017;9(1):136. https://doi. org/10.4300/JGME-D-16-00619.1.

11. ABIM http://www.abim.org/program-directors-administrators/ assessment-tools/mini-cex.aspx. Accessed 1 Mar 2019.

12. Pangaro LA. New vocabulary and other innovations for improving descriptive in-training evaluations. Acad Med. 1999;74(11):1203-7.

13. Seshia SS, Bryan Young G, Makhinson M, Smith PA, Stobart K, Croskerry P. Gating the holes in the Swiss cheese (part I): expanding professor Reason's model for patient safety. J Eval Clin Pract. 2018;24(1):187-97. https://doi.org/10.1111/jep.12847.

14. Veltman LL. Getting to havarti: moving toward patient safety in obstetrics. Obstet Gynecol. 2007;110(5):1146-50.

15. Galvin SL, Buys E. Resident perceptions of service versus clinical education. J Grad Med Educ. 2012;4(4):472-8. https://doi.org/10. 4300/JGME-D-11-00170.1.

16. Rivard LP, Straw, SB. The effect of talk and writing on learning science: an exploratory study. Sci Educ. 2000;84(5):566-93.

17. Gray D, Cozar O, Lefroy J. Medical students' perceptions of bedside teaching. Clin Teach. 2017;14(3):205-10. https://doi.org/10. 1111/tct.12532.

Publisher's Note Springer Nature remains neutral with regard to jurisdictional claims in published maps and institutional affiliations. 